\title{
A finite element model for free surface flows on fixed meshes
}

\author{
A. H. Coppola-Owen and R. Codina ${ }^{*, \dagger}$ \\ Universitat Politècnica de Catalunya, Jordi Girona 1-3, Edifici C1, Barcelona 08034, Spain
}

\begin{abstract}
SUMMARY
In this paper, we present a finite element model for free surface flows on fixed meshes. The main novelty of the approach, compared with typical fixed mesh finite element models for such flows, is that we take advantage of the particularities of free surface flow, instead of considering it a particular case of two-phase flow. The fact that a given free surface implies a known boundary condition on the interface, allows us to solve the Navier-Stokes equations on the fluid domain uncoupled from the solution on the rest of the finite element mesh. This, together with the use of enhanced integration allows us to model low Froude number flows accurately, something that is not possible with typical two-phase flow models applied to free surface flow. Copyright (C) 2007 John Wiley \& Sons, Ltd.
\end{abstract}

Received 22 May 2006; Revised 25 October 2006; Accepted 31 October 2006

KEY WORDS: incompressible free surface flows; stabilized finite element methods; level set; fixed mesh or interface capturing techniques

\section{INTRODUCTION}

Free surface flows are a special kind of flows with moving interface where the influence of one of the fluids over the other one is negligible. Computational fluid dynamics (CFD) approaches for moving interfaces problems are typically categorized into two main groups: Eulerian, fixed mesh or interface capturing techniques [1-7] and Lagrangian, and in the more general case arbitrary Lagrangian Eulerian (ALE), moving mesh or interface tracking techniques [8-10]. The model we will propose is clearly a fixed mesh, interface capturing technique but it is not so obvious whether to classify it as an Eulerian or as a Lagrangian formulation. Actually, the model we propose has

\footnotetext{
*Correspondence to: R. Codina, Universitat Politècnica de Catalunya, Jordi Girona 1-3, Edifici C1, Barcelona 08034, Spain.

†E-mail: ramon.codina@upc.edu

Contract/grant sponsor: Catalan Government

Contract/grant sponsor: The European Social Fund
}

Copyright (C) 2007 John Wiley \& Sons, Ltd. 
two versions: a simplified one which solves the momentum equations in an Eulerian manner and another one that uses an ALE formulation but on a fixed mesh.

In interface capturing techniques, a fixed computational domain is used together with an interface function to capture the position of the interface. The interface is captured within the resolution of the fixed mesh and the boundary conditions at the interface are somehow approximated. In interface tracking techniques, the mesh is updated in order to track the interface. The simplest approach is to deform the mesh without changing its topology, but this is possible only for very simple flows. As the flow becomes more complex and unsteady, remeshing is required, and consequently the projection of the results from the old to the new mesh is needed. In 3D calculations, these operations can introduce costs that can render moving mesh techniques unfeasible. This is the main reason why we prefer to use fixed mesh methods. Both approaches can deal with two-phase or free surface flows. In moving mesh simulations, it is very common to see real free surface simulations where only one fluid is modelled and the mesh follows the movement of the interface. On the other hand, in finite element fixed mesh simulations free surface flows are typically treated as a particular case of two-phase flows where the second fluid is air or some pseudo-fluid with a density and viscosity much smaller than the first fluid.

The typical Eulerian approach can work well in a great number of situations but in some cases it can fail miserably. For example, in two fluid flows when gravity forces are much more important than inertial effects. This problem has been analysed in a previous paper [11]. The main problem is

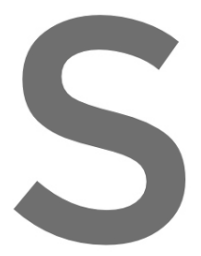
that since we are using dient is discontinuous shape functions and gradient and the grav spoil the simulation. shape functions in the
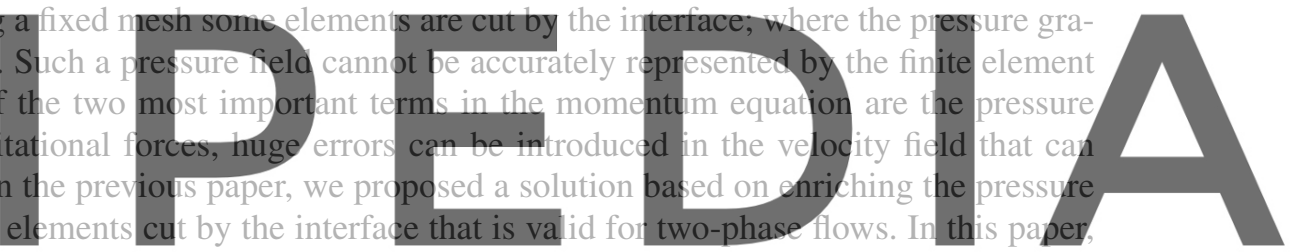

we will propose an alternative solution that is valid only for free surface flows. This might seem

of free surface flows deserves special attention due to the fact that a great proportion of two-phase flows of practical interest are free surface fiows. Since the effect of surface tension is negligible in the flows we are interested in we will not take such effects into account in the model we propose.

The solution we propose in this paper is to model free surface flows on fixed grids in a similar way to the one used for two-phase flows but modelling in principle only the part of the domain filled by the liquid. Since we are using a fixed grid method we will have elements that are totally filled by liquid and others only partially. The main question is what to do in partially filled elements. What we propose to do is to integrate only in the part filled by the fluid. In order to do so we use special integration rules in elements cut by the front that have been introduced in the previous paper for two-phase flows with enriched pressure shape functions [11]. This will allow us to impose the correct boundary conditions on the free surface, the key to the success of the method.

Fixed mesh methods generally share two basic steps. One where the motion in both phases is found as the solution of the Navier-Stokes equations for one-phase flow with variable properties. In the second one, an equation for an interface function that allows to determine the position of the interface, and thus the properties to be assigned in the previous step, is solved. As we have already mentioned, the model we will present has two versions, one that solves the Navier-Stokes equations in an Eulerian manner and the other one that uses an ALE formulation. In both versions, a level set function is used to determine the position of the interface. 
As we have already mentioned, two-phase flow level set models are much more common than the free surface single-phase flow level set model we present in this paper. A recent single-phase level set model can be found in [12]. The main difference with our model is that it uses a convective extension to obtain the velocities at the previous time steps for the grid points in air while we use a fixed meshes ALE approach. Moreover, in the cited paper a finite volume structured grid is used while we use an unstructured finite element mesh.

Since the Navier-Stokes equations in an Eulerian form can be seen as a particular case of the ALE form when the domain velocity is zero, we will in the next section present briefly the ALE version of the Navier-Stokes equations, and its space and time discretization. Interface capturing techniques will be discussed in Section 3. In Section 4, we will present the free surface model that uses the fixed mesh ALE formulation. In Section 5, a simplified Eulerian version of the free surface model will be presented. In Section 6, we will analyse three numerical examples that have already been used in the paper for two-phase flows with enriched pressure shape functions [11]. We will demonstrate that the free surface model, in any of its two versions, allows to obtain results that are similar or better to the ones obtained with enriched pressure shape functions and much better than those obtained with a typical two-phase Eulerian model.

\section{FINITE ELEMENT APPROXIMATION OF THE NAVIER-STOKES EQUATIONS}
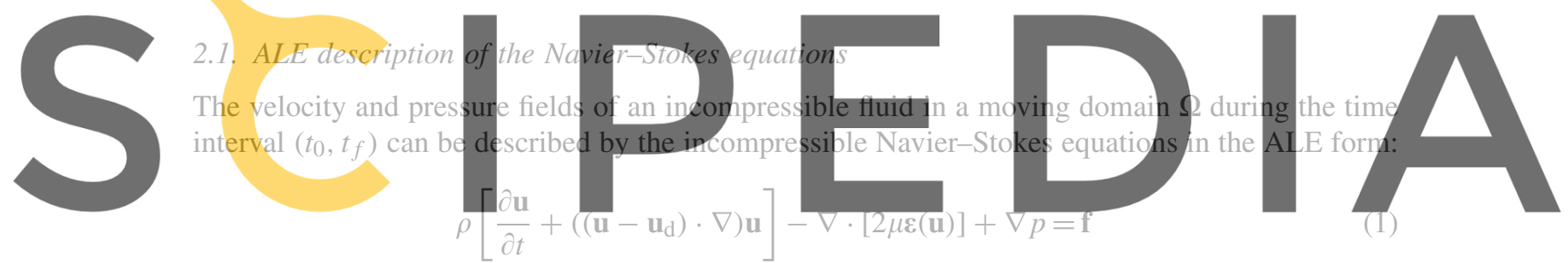

Register for free at https//www.scipedia.com. to download the version without the 2 watermark

where $\rho$ is the density, $u$ the velocity field, $\mathbf{u}_{\mathrm{d}}$ the domain velocity, $\mu$ the dynamic viscosity, $p$ the pressure, $\boldsymbol{\varepsilon}(\cdot)$ the symmetric gradient operator and $\mathbf{f}$ the vector external body forces, which includes the gravity force $\rho \mathbf{g}$ and buoyancy forces, if required.

Let $\boldsymbol{\sigma}$ be the stress tensor and $\mathbf{n}$ the unit outward normal to the boundary $\partial \Omega$. Denoting by an over-bar prescribed values, the boundary conditions to be considered are:

$$
\mathbf{u}=\overline{\mathbf{u}} \quad \text { on } \Gamma_{\mathrm{du}}, \quad \mathbf{n} \cdot \boldsymbol{\sigma}=\mathbf{0} \quad \text { on } \Gamma_{\mathrm{nu}}, \quad \mathbf{u} \cdot \mathbf{n}=0, \quad \mathbf{n} \cdot \boldsymbol{\sigma} \cdot \mathbf{g}_{1}=0, \quad \mathbf{n} \cdot \boldsymbol{\sigma} \cdot \mathbf{g}_{2}=0 \quad \text { on } \Gamma_{\mathrm{mu}}
$$

for $t \in\left(t_{0}, t_{f}\right)$. Vectors $\mathbf{g}_{1}$ and $\mathbf{g}_{2}$ (for the three-dimensional case) span the space tangent to $\Gamma_{\mathrm{mu}}$. Observe that $\Gamma_{\mathrm{du}}$ is the part of the boundary with Dirichlet velocity conditions, $\Gamma_{\mathrm{nu}}$ the part with Neumann conditions (prescribed stress, for example, the free surface) and $\Gamma_{\mathrm{mu}}$ the part with mixed conditions. These three parts do not intersect and are a partition of the whole boundary $\partial \Omega$. Proper initial conditions have to be applied to the problem.

\subsection{Space and time discretization}

Let $\mathbf{V}_{h}^{*}$ and $Q_{h}^{*}$ be the finite element spaces to interpolate vector and scalar functions, respectively, constructed in the usual manner and using the same interpolation from a finite element partition $\Omega=\bigcup \Omega^{e}, e=1, \ldots, n_{\mathrm{el}}$, where $n_{\mathrm{el}}$ is the number of elements. In the case of the free surface 
formulation we are proposing in this paper that $\Omega$ is not formed by all the elements but only by those full of liquid and by the liquid part of elements cut by the interface, as determined by the level set function $\psi$. The complete details will be discussed in Section 4. From these spaces one can construct the subspaces $\mathbf{V}_{h, u}$ and $Q_{h}$ for the velocity and the pressure, respectively. The former incorporates the Dirichlet conditions for the velocity components and the latter has one pressure fixed to zero if the normal component of the velocity is prescribed on the whole boundary. The space of velocity test functions, denoted by $\mathbf{V}_{h}$, is constructed as $\mathbf{V}_{h, u}$ but with functions vanishing on the Dirichlet boundary. Let also $\theta$, with $0<\theta \leqslant 1$, be the parameter of the trapezoidal rule for time discretization and $\delta t$ the time step size, chosen constant for simplicity. The algorithmic solution to the problem will be computed at $t^{n}=n \delta t, n=1,2, \ldots$.

The algebraic version of the subgrid scale stabilization method, referred to ASGS in the following [13] is used to deal with convection-dominated fows and to circumvent the well known div-stability restriction for the velocity and pressure finite element spaces [14], allowing in particular for equal interpolation of both unknowns. The ASGS monolithic discrete problem associated with the Nayier-Stokes equations (1)-(2), discretizing in time using the generalized trapezoidal rule, and linearizing the convective term using a Picard scheme, can be written as follows: given a velocity $\mathbf{u}_{h}^{n}$ at time $t^{n}$, a domain velocity $\mathbf{u}_{\mathrm{d}, h}^{n+\theta}$ at time $t^{n+\theta}$, and a guess for the unknowns at an iteration $i-1$ at time $t^{n+1}$, find $\mathbf{u}_{h}^{n+\theta, i} \in \mathbf{V}_{h, u}$ and $p_{h}^{n+\theta, i} \in Q_{h}$, by solving the discrete variational problem
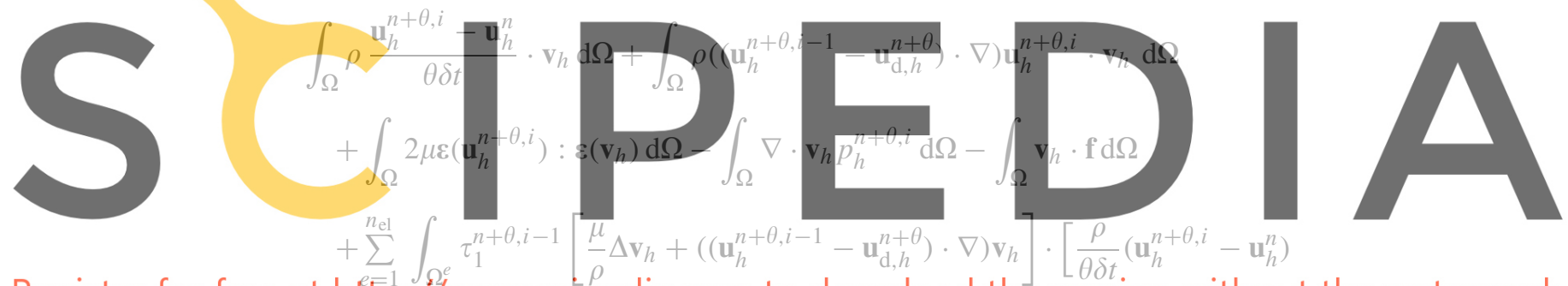

Register for free at hteps $/ \%$ www.scipedia.com to download the version without the watermark

$$
\begin{aligned}
& \left.-\nabla \cdot\left[2 \mu \varepsilon\left(\mathbf{u}_{h}^{n+\theta, i}\right)\right]+\rho\left(\left(\mathbf{u}_{h}^{n+\theta, i-1}-\mathbf{u}_{\mathrm{d}, h}^{n+\theta}\right) \cdot \nabla\right) \mathbf{u}_{h}^{n+\theta, i}+\nabla p_{h}^{n+\theta, i}-\mathbf{f}\right] \mathrm{d} \Omega \\
& +\sum_{e=1}^{n_{\mathrm{el}}} \int_{\Omega^{e}} \tau_{2}^{n+\theta, i-1}\left(\nabla \cdot \mathbf{v}_{h}\right)\left(\nabla \cdot \mathbf{u}_{h}^{n+\theta, i}\right) \mathrm{d} \Omega=0 \quad \forall \mathbf{v}_{h} \in \mathbf{V}_{h} \\
& \int_{\Omega} \rho q_{h} \nabla \cdot \mathbf{u}_{h}^{n+\theta, i}+\sum_{e=1}^{n_{\mathrm{el}}} \int_{\Omega^{e}} \tau_{1}^{n+\theta, i} \nabla q_{h} \cdot\left[\frac{\rho}{\theta \delta t}\left(\mathbf{u}_{h}^{n+\theta, i}-\mathbf{u}_{h}^{n}\right)-\nabla \cdot\left[2 \mu \boldsymbol{\varepsilon}\left(\mathbf{u}_{h}^{n+\theta, i}\right)\right]\right. \\
& \left.\quad+\rho\left(\left(\mathbf{u}_{h}^{n+\theta, i-1}-\mathbf{u}_{\mathrm{d}, h}^{n+\theta}\right) \cdot \nabla\right) \mathbf{u}_{h}^{n+\theta, i}+\nabla p_{h}^{n+\theta, i}-\mathbf{f}\right] \mathrm{d} \Omega=0 \quad \forall q_{h} \in Q_{h}
\end{aligned}
$$

for $i=1,2, \ldots$ until convergence, that is to say, until $\mathbf{u}_{h}^{n+\theta, i-1} \approx \mathbf{u}_{h}^{n+\theta, i}$ and $p_{h}^{n+\theta, i} \approx p_{h}^{n+\theta, i-1}$ in the norm defined by the user.

The parameters $\tau_{1}$ and $\tau_{2}$ are chosen in order to obtain a stable numerical scheme with optimal convergence rates (see [13] and references therein for details). They are computed within each element domain $\Omega^{e}$. We take them as

$$
\tau_{1}=\frac{\rho\left(h^{e}\right)^{2}}{4 \mu+2 \rho h^{e}\left|\mathbf{u}_{a}^{e}\right|} \quad \text { and } \quad \tau_{2}=\mu+\frac{1}{2} \rho h^{e}\left|\mathbf{u}_{a}^{e}\right|
$$


where $h^{e}$ and $\left|\mathbf{u}_{a}^{e}\right|$ are a typical length and an ALE velocity norm of element $e$, respectively. The ALE velocity is defined as $\mathbf{u}_{a}=\mathbf{u}-\mathbf{u}_{\mathrm{d}}$.

Once the algorithm has produced a converged solution, the velocity field at $t^{n+1}$ can be updated from the velocity at $t^{n+\theta}$ by using the relation $\mathbf{u}^{n+1}=\left[\mathbf{u}^{n+\theta}-(1-\theta) \mathbf{u}^{n}\right] / \theta$.

\subsection{Eulerian Navier-Stokes equations for one or two fluids}

The Eulerian description can be obtained as a particular case of the ALE Navier-Stokes equations where the domain velocity is zero. For the one fluid case the equations can then be discretized exactly as described for the ALE formulation taking into account that $\mathbf{u}_{\mathrm{d}}=\mathbf{0}$.

In the two fluid case the domain $\Omega$ is formed by $\Omega_{1} \cup \Omega_{2}$, where $\Omega_{1}$ indicates the part of $\Omega$ occupied by fluid number 1 and $\Omega_{2}$ indicates the part of $\Omega$ occupied by fluid number 2 . The extent of $\Omega_{1}$ and $\Omega_{2}$ is given by the level set function $\psi[1]$.

The equations remain unaltered from the Eulerian one fluid case, except for the fact that the density, velocity, dynamic viscosity and pressure are defined as

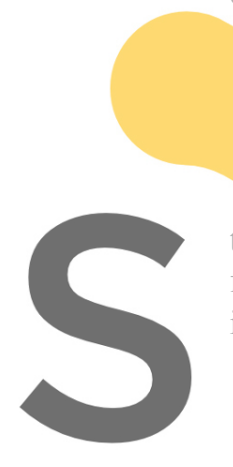

$$
\mathbf{u}, p, \rho, \mu= \begin{cases}\mathbf{u}_{1} p_{1}, \rho_{1}, \mu_{1}, & \mathbf{x} \in \Omega_{1} \\ \mathbf{u}_{2} p_{2}, \rho_{2}, \mu_{2}, & \mathbf{x} \in \Omega_{2}\end{cases}
$$

In the free surface formulation we present in this paper $\Omega=\Omega_{1}$ and $\Omega_{2}$ does not exist. Nevertheless, we will still u fluid Eulerian simulation in Sections 4 and 5.
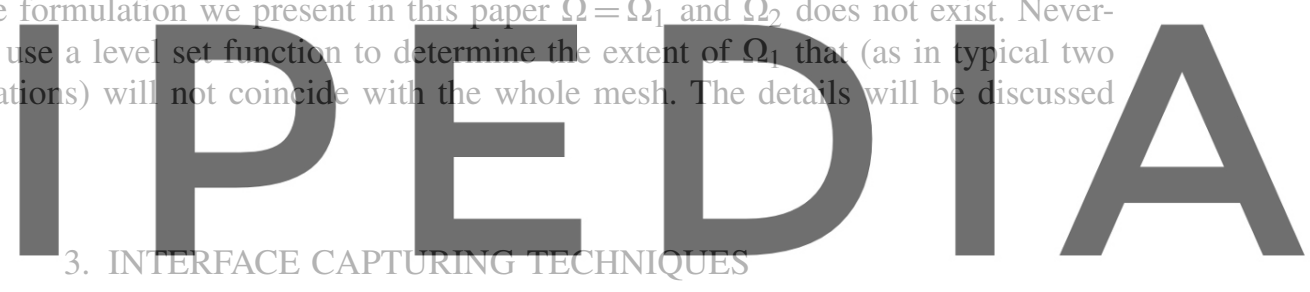

Register fob.freeriatihttop//www.scipedia.com to download the version without the watermark

Typical fixed mesh methods generally share two basic steps. One where the motion in both phases is found as the solution of the Navier-Stokes equations for a one phase flow with variable properties, and the other one where an equation for an interface function that allows to determine the position of the interface, and thus the properties to be assigned in the previous step, is solved. The different approaches differ mainly in the method used to determine the position of the interface, but differences can also be found in the way fluid properties are approximated close to the interface.

Referring to the evolution of the fluid interface, we update it using the so-called level set method (see [1-3] for an overview), also called pseudo-concentration technique [4] which is very similar to the volume of fluid (VOF) technique [5]. This formulation has been widely used to track free surfaces in mould filling (see, for example, [6,7], among other references) and other metal forming processes.

The level set method leads to a partial differential transport equation, the solution of which determines the position of the free surface as an isovalue of the unknown of this equation, which we will call $\psi$. This equation is hyperbolic and therefore it is necessary to use a stabilized finite element method to solve it.

In the formulation we present in this paper we only simulate one fluid bounded by a moving free surface. As we have already mentioned, the fluid domain where the Navier-Stokes equations are solved occupies only part of the fixed mesh. The level set function that determines the extent 
of the fluid domain is nevertheless solved over the whole mesh. In the part of the mesh not filled by liquid a velocity is needed to transport the level set function. The way in which it is obtained will be described in the next two sections.

\subsection{Implementation of the level set method}

The basic idea of the level set method is to define a smooth scalar function, say $\psi(\mathbf{x}, t)$, over the computational domain $\Omega$ that determines the extent of subdomains $\Omega_{1}$ and $\Omega_{2}$. For instance, we may assign positive values to the points belonging to $\Omega_{1}$ and negative values to the points belonging to $\Omega_{2}$. The position of the fluid front will be defined by the iso-value contour $\psi(\mathbf{x}, t)=0$. The evolution of the front $\psi=0$ in any control volume $V_{t} \subset \Omega$ which is moving with a divergence free velocity field $u$ leads to:

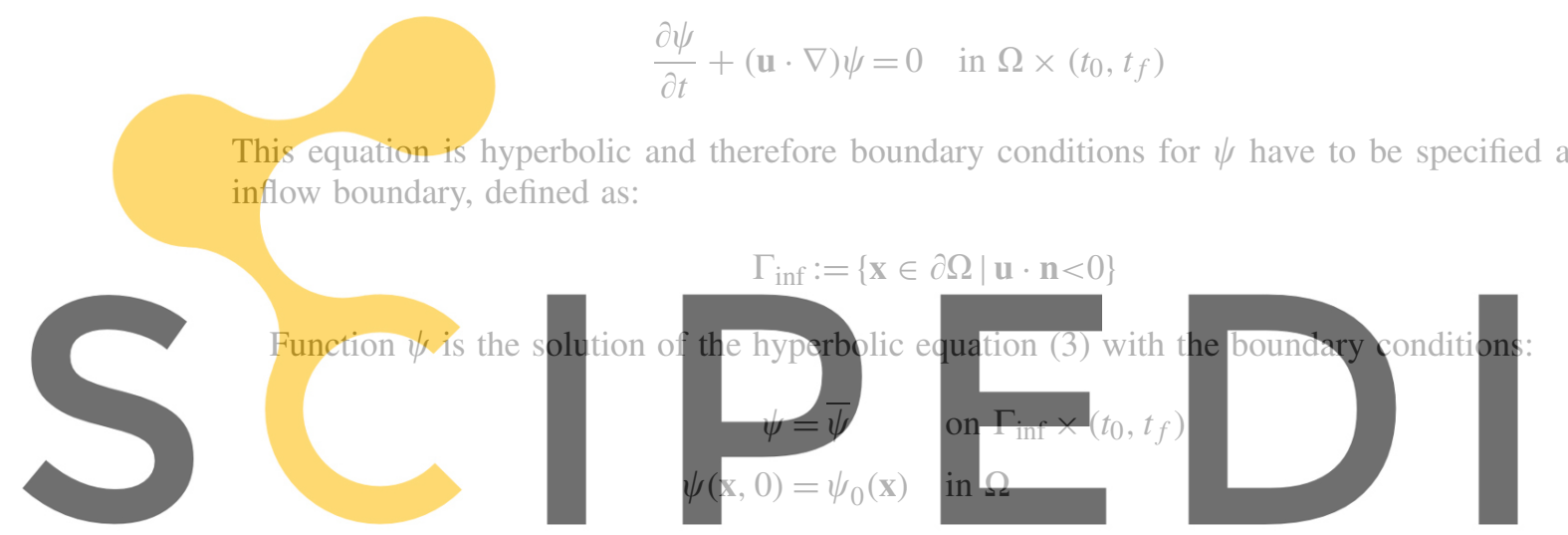

This equation is hyperbolic and therefore boundary conditions for $\psi$ have to be specified at the inflow boundary, defined as:

\section{Register for f}

The initial condition $\psi_{0}$ is chosen in order to define the initial position of the fluid front to be opifileecatiht thos
inflow boundary.

Due to the pure convective type of the equation for $\psi$, we use the SUPG technique for the spatial discretization. Again, the temporal evolution is treated via the standard trapezoidal rule.

For the numerical solution of the level set equation it is preferable to have a function without large gradients. Since the only requirement such a function must meet is $\psi=0$ at the interface, a signed distance function $(|\nabla \psi|=1)$ is used. Under the evolution of the level set equation, $\psi$ will not remain a signed distance function and thus needs to be reinitialized. This can be achieved by redefining $\psi$ for each node of the finite element mesh according to the following expression:

$$
\psi=\operatorname{sgn}\left(\psi^{0}\right) d
$$

where $\psi^{0}$ stands for the calculated value of $\psi, d$ is the distance from the node under consideration to the front, and $\operatorname{sgn}(\cdot)$ is the signum of the value enclosed in the parenthesis.

Since the objective of this paper is to analyse the improvements that can be obtained in the solution of the Navier-Stokes equations and not to optimize the solution of the level set equations, a very simple algorithm has been used to calculate the distance $d$. Using linear elements, the free surface is approximated by triangular planes $p$ (lines in two-dimension). Then the perpendicular distance $d_{i} p$ of each grid point $i$ to each plane $p$ can be computed. The minimum distance from each nodal point to the planes is the required distance between the point and the front $\left(d_{i}=\min _{p}\left\{d_{i} p\right\}\right)$. 


\section{FM-ALE-FREE SURFACE MODEL}

In the previous sections we have described some techniques that are commonly used in the simulation of free surface or two-phase flows. Now we will present the way in which we combine them to give rise to a new free surface model on fixed meshes. We will start from a typical Eulerian simulation for a two-phase flow and describe the way in which our model departs from it.

As has already been mentioned, a typical Eulerian simulation includes two main steps. One which solves Eulerian two fluid Navier-Stokes equations and the other one which determines the interface position. Both are solved over the entire mesh. When free surface flow is considered, the properties used in the second fluid are much smaller than those in the main fluid. The model we propose solves the Navier-Stokes equations only on one fluid bounded by a moving free surface whose position, as in the typical model, is determined by the level set function. Therefore the domain $\Omega$ where we solve the Navier-Stokes equations does not extend over the whole mesh, but only over totally filled elements and over the filled part of elements cut by the interface. This is an important difference with typical finite element simulations (Eulerian, Lagrangian or ALE) where the domain where the flow is simulated extends over the whole mesh. In order to be able to use a domain that includes portions of elements, special integration rules have to be used. The integration rule we use here has been presented in a previous paper [11] where we deal with pressure enrichment for two-phase flows and consist in dividing the elements cut by the front into subelements only for integration purposes. In a finite element setting the free surface boundary condition is a natural it correctly even if the imposing the correct mesh formulation is o of both worlds (Eulerian
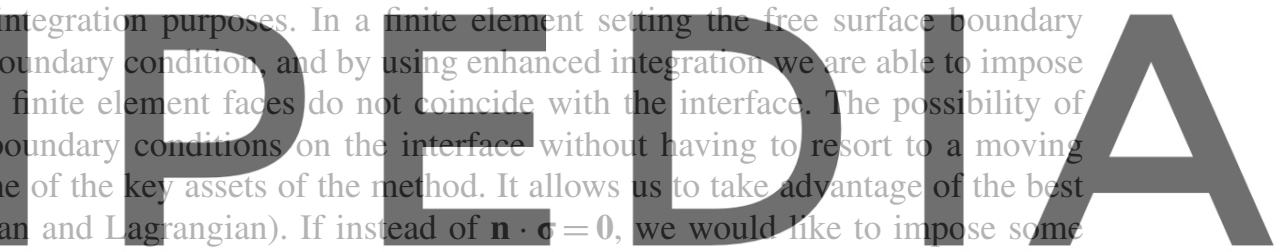

prescribed value $\mathbf{n} \cdot \boldsymbol{\sigma}=\mathrm{t}$ on the free surface, the procedure would be slightly more complicated.

cut element, the front (as defined by the level set) would be divided into triangles where the usual surface integration rules would be used. Thus, one would be able to build the term corresponding to the Neumann boundary condition applied on the interface, $\int_{\Gamma} \mathbf{v} \cdot \mathbf{t} \mathrm{d} \Gamma$.

The second main difference with typical fixed mesh interface simulations is that we use an ALE description when solving the Navier-Stokes equations. The particularity of our ALE description is that it is used on a fixed mesh. It is called FM-ALE and has been introduced in a paper on lost foam casting [15]. In this paper we extend it to free surface flows. The idea behind the FM-ALE method is quite simple; if one wants to simulate a moving domain on a fixed grid using an ALE description what one must do is to project the results obtained on the deformed domain on the portion of the fixed mesh occupied by the fluid at each time step.

The method we propose consist of three main steps in going from time $n$ (where velocities and interface position are given) to time $n+1$ :

(1) Find the interface position at step $n+1$ by solving the level set equation. An explicit treatment is used in this step, that is, the velocities at step $n$ are used.

(2) Obtain the domain velocity and fluid velocity at time $n$ on the new domain determined in the previous step.

(3) Solve the Navier-Stokes equations on the new domain using an ALE description. 


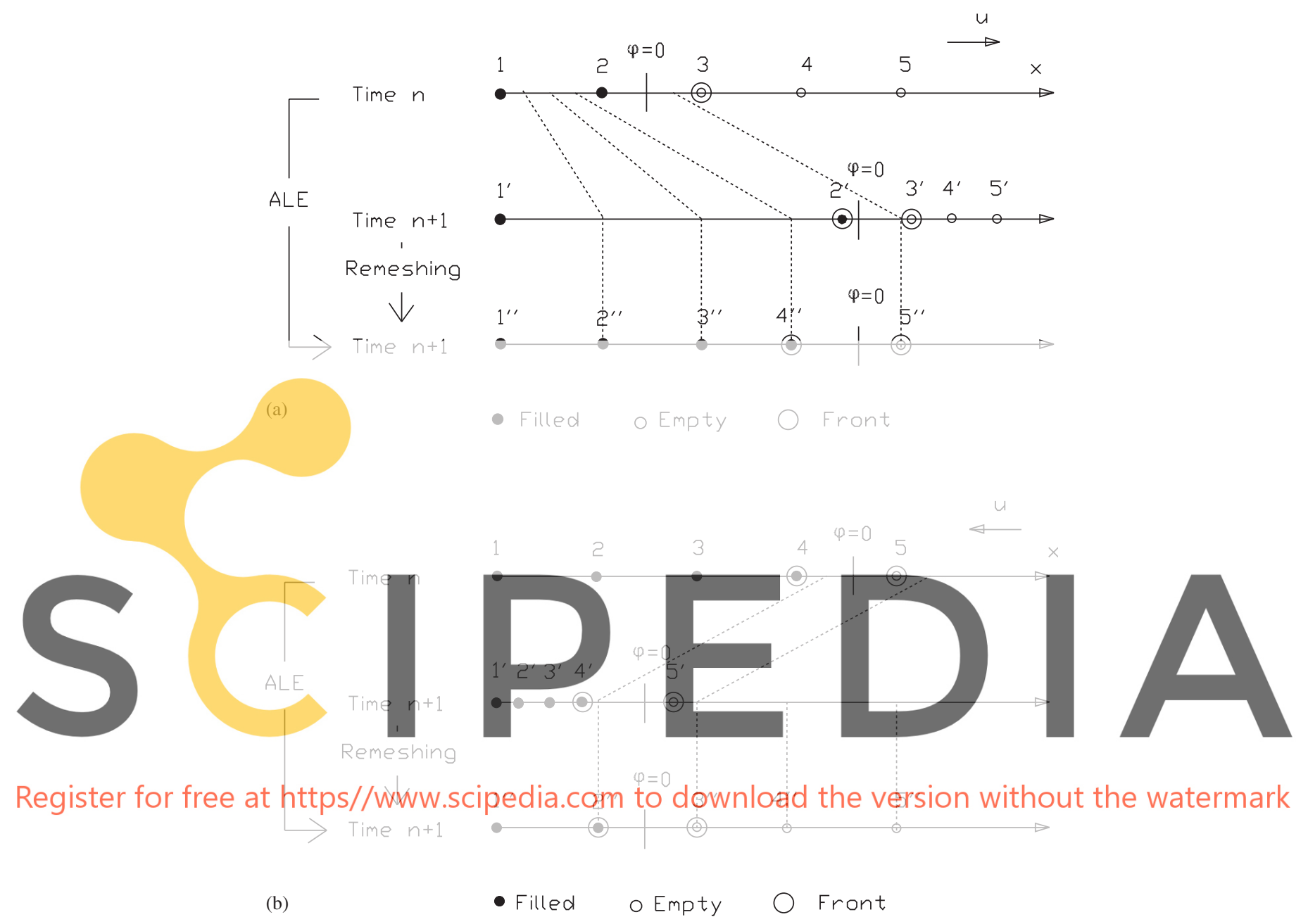

Figure 1. One-dimensional FM-ALE example: (a) positive velocity; and (b) negative velocity.

The way in which to solve the first and third step have already been discussed. We will now describe the way in which to deal with the second step. The key point here is how to define the domain velocity at time $n+1$. It will depend on the fluid velocity at time $n$ and on the interface position at times $n$ and $n+1$. We will use the one-dimensional example shown in Figure 1 to explain how it is obtained. Contrary to what happened in the original FM-ALE paper [15] where the domain could only expand, in a free surface simulation the domain can either expand or contract.

The domain velocity at time $n+1$ will depend on the fluid velocity at time $n$ and on the level set function at times $n$ and $n+1$. In order to describe the method we will classify the nodes into empty and filled nodes. The nodes belonging to cut elements will be additionally described as 
front nodes. The domain velocity will be set to zero at nodes that belong to the fluid but not to the front at times $n+1$ and $n$ on the fixed mesh. That is the case of node 1 in Figures 1(a) and (b), where Figure 1(a) represents the case where the domain has a positive $x$ velocity (it is expanding) and Figure 1(b) represents the case where the domain has a negative $x$ velocity (it is contracting). The domain velocity will be set to

$$
\mathbf{u}_{\mathrm{d}}^{n+1}=\mathbf{n}\left(\mathbf{u}^{n} \cdot \mathbf{n}\right)
$$

at nodes that belong to the front or to the air at time $n$. The normal direction $\mathbf{n}$ is obtained from the level set function at each node as

$$
\mathbf{n}=\frac{\nabla \psi^{n+1}}{\left|\nabla \psi^{n+1}\right|}
$$

Such is the case of nodes 2-5 in Figure 1(a) and nodes 4 and 5 in Figure 1(b). Using the previous conventions, in the expanding case (Figure 1(a)) the domain velocity is defined on all the nodes. In the contracting case it is not be defined at nodes 2 and 3. There it is obtained by solving

$$
\Delta \mathbf{u}_{\mathrm{d}}=0
$$

with the boundary conditions defined previously. Once the domain velocities have been obtained, the displacements in one time step are simply $\delta \mathbf{x}=\boldsymbol{u}_{\mathrm{d}} \delta t$. They allow us to obtain the deformed

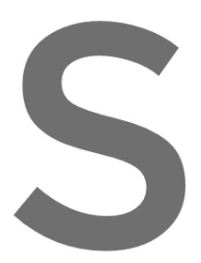
mesh, shown in the middle line. Finally, the m
be obtained on the fixed mesh at time $n+1$ (bot
the deformed mesh as shown by dotted lines. T
Navier-Stokes equations in ALE form.
If the free surface moves by a large amount become invalid. In that case a smaller time step methods. Of course, progressive distortion does not make sense in our approach.

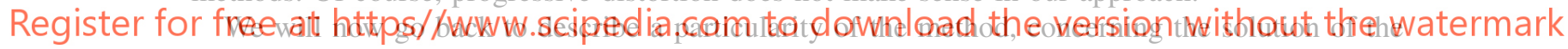

level set equation. We have already mentioned the level set equation is solved over the whole mesh and therefore the velocities on the region formed by non-filled elements must be defined in some way. This is not a big problem, since in theory the only velocities needed to transport the interface position are those on the interface defined by $\psi=0$. Several options to define extension velocities that allow us to obtain a velocity field on the whole mesh from the velocities on the interface can be found in the literature [3]. The approach we will use is a relatively simple one [15]: on the part of the mesh formed by empty elements, a stationary Stokes problem will be solved. On the air front nodes the velocities obtained when solving the fluid will be used as Dirichlet boundary conditions. The boundary conditions used when solving for the extension velocities have no influence on the resulting fluid flow and will be taken as in a typical Eulerian two fluid simulation. The fluid properties used when solving the Stokes problem in the empty region are those of the fluid.

From the description of the FM-ALE formulation it is seen that the major differences with respect to the classical ALE approach are the following:

- Given a position of the fluid front on the fixed mesh, elements cut by the front are split into subelements (only for integration purposes), so that the front coincides with the edges of the subelements. This allows to prescribe $\mathbf{n} \cdot \boldsymbol{\sigma}=\mathbf{0}$ as boundary condition. In fact, it is only necessary to modify the integration rule, as explained earlier. Once this is done, the flow equations can be solved. 

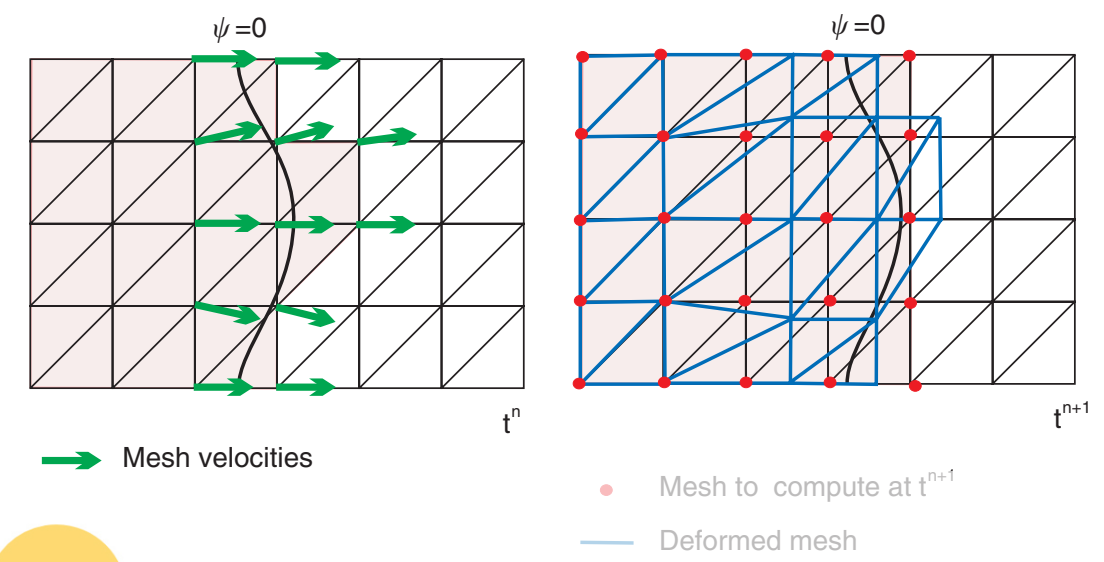

Figure 2. Two-dimensional FM-ALE schematic.

- After deforming the mesh from one time step to the other using classical ALE procedures, results are projected back to the original mesh (through interpolation, projection with restrictions as explained in [16] or any other technique).
of the material points at the free suface as in a classical ALE method. Note that the previous
step implies that front nodes cannot be trackec.
A schematic of the FM-ALE approach for frec surface flows is presented in Figure 2 .
n essence, this formulation is the same as the one presented in [15] with two major differences:

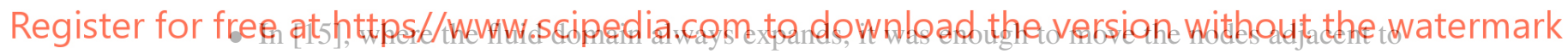

the front. In the present model, since the domain can also contract, a more general definition for the mesh velocities had to be adopted. The mesh velocities at time $n+1$ depend on the fluid velocities at time $n$ and on the level set function both at times $n$ and $n+1$. When the domain contracts some nodes of the fluid domain are deformed by solving Equation (5) with boundary conditions determined from the rules used to define the mesh velocity on the rest of the nodes.

- In the lost foam model of [15], the front velocity is given and the pressure is unknown. In the present free surface model, the front velocity is unknown and the traction is given $(\mathbf{n} \cdot \boldsymbol{\sigma}=\mathbf{0})$.

\section{EULERIAN SIMPLIFIED FREE SURFACE MODEL}

In the previous section we have presented a version of the free surface model that solves the Navier-Stokes equations on the fluid only using an ALE description. In this section we will introduce a simpler version; the main difference being that an Eulerian description is used. The model will therefore be more closely related to the typical Eulerian two-phase model. Nevertheless the key element of the model, solving the Navier-Stokes equations only on the fluid region $\Omega$ (totally filled element plus filled part of cut elements), will remain unaltered. 
The way to understand this model is to think of it as a typical two-phase flow model, with a second fluid with negligible properties and where the null traction boundary condition has been added on the interface. This boundary condition allows us to uncouple the solution of the fluid from that of the air making the problem much simpler. We will often refer to the second fluid as air even if it may be some other fluid. Suppose that we are solving a typical two-phase flow model using enhanced integration (see Section 4) in the elements cut by the front. When one looks at the discrete momentum equation it is clear that the contribution to the matrix and right-hand side corresponding to the transient, convective, diffusive and external force terms will tend to zero in the air elements and in the part of the cut elements filled by air. Thus all the terms in the momentum equation, except the pressure gradient, tend to the same value if one uses the free surface model we propose or a two-phase model with negligible second fluid. In order to interpret what happens with the pressure term when using the free surface methodology as compared to the two-phase case, one has to observe that if all other terms tend to zero in the region not occupied by the liquid then the solution there would have a null pressure gradient. If one introduced such information $a$ priori in the two-phase case, then the systems resulting from the momentum equation would be identical when the fluid properties tend to zero. The problem with the two-phase model, when used with the typical finite element functions, is that they cannot represent a pressure gradient that is zero in one part of the element and different from zero in the other. As we have already said, since the velocity and pressure are coupled, the impossibility of accurately representing the pressure can introduce errors in the velocity that can render the solution meaningless. Enriching the pressure shape functions is a way to solve such problem [11], the free surface formulation we present here is another solution, perhaps simpler. They will be compared numerically in Section 6.

Finally, one also has to look at the continuity equation. The contribution to the system matrix would be different if one uses Equation (2). But it could be replaced by

$$
\rho \nabla \cdot \mathbf{u}=0
$$

From the continuum point of view both equations are equivalent. The numerical approximation of Equation (6) would lead to identical system matrices if one uses the free surface model or the two-phase model. Using Equation (6) can be seen as weighting the incompressibility constraint depending on the density of the fluid. In the free surface case what we are doing is only imposing incompressibility in the region where fluid exists. It is a pretty logical hypothesis.

One important point to remark is that as we are using enhanced integration we are able to integrate only in the domain filled by liquid and thus we can impose the Neumann boundary condition corresponding to the free surface $(\mathbf{n} \cdot \boldsymbol{\sigma}=\mathbf{0})$ accurately exactly where the interface is located according to the level set function. This is a key point for the success of the method. On the other hand, since we are only simulating one fluid we do not have a discontinuous pressure gradient and therefore no special shape functions are needed.

As in the FM-ALE free surface model described in the previous section, the level set equation must be solved on the whole mesh, not only in the liquid region. In order to reduce computational labour the level set equation could be solved only in a small region close to the interface using the narrow-band approach [17]. Once again, the velocity must be defined in the empty region somehow, in order to transport the level set function. In this case the solution adopted is very similar to the one used in the previous section except for the fact that instead of solving the steady Stokes equations with fluid properties we will use the transient Navier-Stokes equations with air (or some pseudofluid) properties. The boundary conditions will be the same as in the FM-ALE model. The reason for this choice is that we are justifying this model based on what happens in the 
Eulerian two-phase case and therefore it seems logical to solve for the air as similarly as possible as done in that model. We would nevertheless like to point out that the way in which the air is solved is not of great importance.

Contrary to what happens in the FM-ALE model where the velocities in the empty region are only needed to transport the level set, in the Eulerian free surface model there is another reason for obtaining those velocities. Since the fluid domain is moving and the mesh is fixed there will be nodes that in step $n+1$ belong to the fluid but in the previous step belonged to the empty region. In such nodes we will use the velocities calculated for the empty region when modelling the transient term. The validity of such approach is justified once again pointing out that it is what happens in the case of a two-phase flow when the properties of the second fluid tend to zero.

\section{NUMERICAL EXAMPLES}

In this section we present three numerical examples where one can appreciate the benefits the proposed formulation can provide compared to a typical two-phase flow model applied to free surface flow. The examples have been borrowed from a previous paper [11] where they have been used to test an Eulerian two-phase flow model with enriched pressure shape functions. The results obtained with the free surface model will be compared with the results obtained with a typical two-phase flow model and also with the enriched pressure model presented in the previous paper.

\subsection{Two-fluid cavity}

The first example, called the two-fluid cavity, is a square domain filled with equal amounts of two different density fluids and a fixed horizontal velocity $(0.1 \mathrm{~m} / \mathrm{s})$ on the bottom wall. In the free surface case only the lower half of the mesh is filled by fluid. The walls are supposed frictionless and the top face is left open. It is a very simple example but it can be representative of the numerical problems that can appear in much more complex problems.

For comparison purposes, the same three two-dimensional unstructured triangular meshes as in [11] were used (see Table I). The square domain has a side length $L=10 \mathrm{~m}$. The material properties used (SI units) are $\rho_{1}=1000, \mu_{1}=10$ for the fluid on the bottom. The viscosity of the bottom fluid is 1000 times the viscosity of water so as to obtain a relatively low Reynolds number $(R e=100)$ in order to avoid unnecessary complications. The simulations were run for $100 \mathrm{~s}$ with a $0.5 \mathrm{~s}$ time step size. In the two-phase flow simulations the properties of the second fluid used are 100 times smaller than those of the first fluid so as to be in a free surface case. They differ from those used in paper [11] where they were similar to the properties of the first fluid. With such density difference the problem turned out to be more difficult to solve and therefore for this example the acceleration of gravity is reduced to $g=1.0$. In the rest of the examples it is $g=10.0$. The Froude number for this example is $F r=0.001$.

Table I. Meshes used for the two-fluid cavity flow.

\begin{tabular}{lrrc}
\hline Mesh & Nnode & Nelem & Elem length \\
\hline Coarse & 128 & 214 & 1.0 \\
Medium & 472 & 862 & 0.5 \\
Very fine & 11615 & 22828 & 0.1 \\
\hline
\end{tabular}



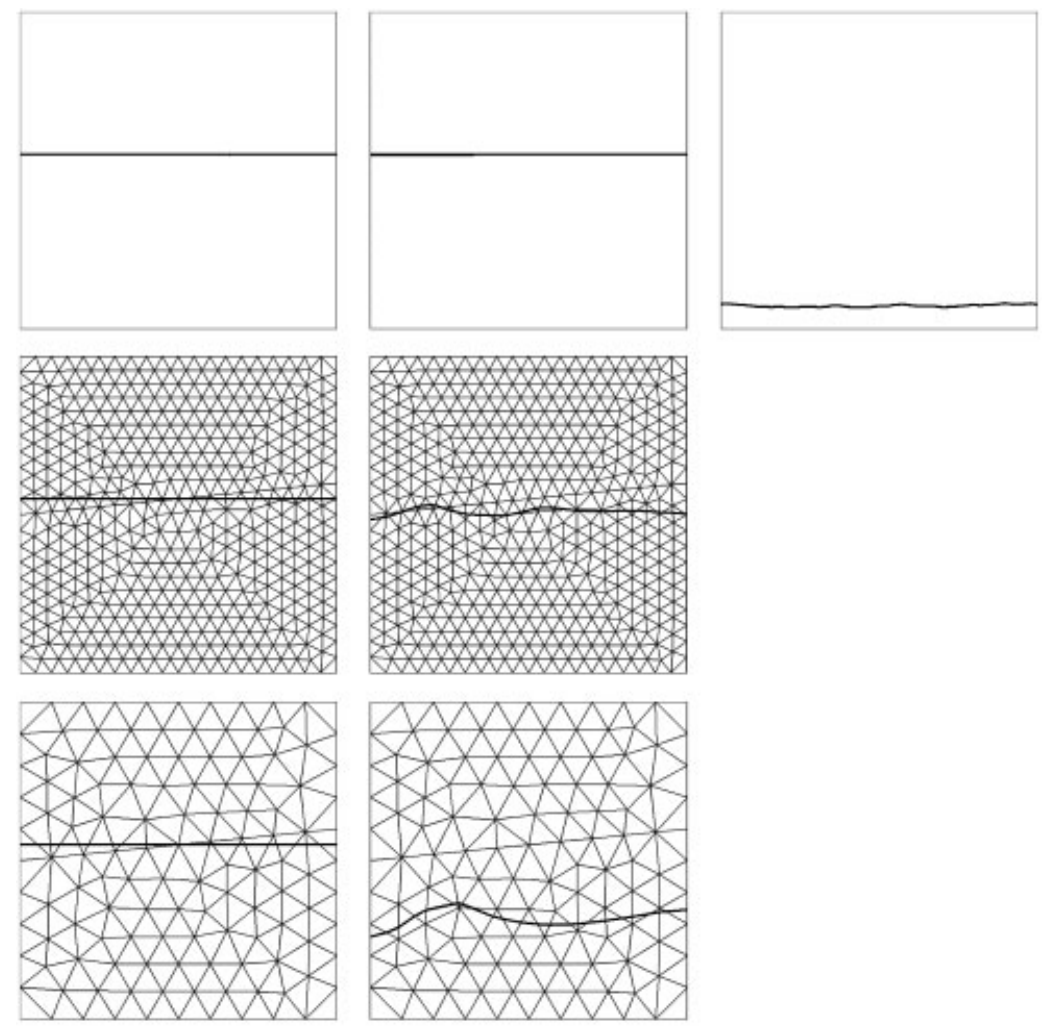

Free Surface

FM-ALE model

Enriched pressure two-phase model
Normal two-phase model

Figure 3. Shape of the interface for the different meshes and numerical models at $t=100 \mathrm{~s}$ (top: very fine mesh, middle: medium mesh, bottom: coarse mesh).

In Figure 3 we show the shape of the interface for the three meshes with three different models: the FM-ALE free surface model, the enriched pressure two-phase model [11] and the normal two-phase model.

Using the finest mesh the free surface model and the enriched pressure two-phase model give nearly the same result. The normal two-phase model fails to predict the correct interface position. There is an erroneous fall of the interface created by an incorrect velocity field as shown in Figure 4. Despite that we have not got physical measurements, the two correct solutions obtained with this mesh can be taken as a reference against which we can compare the results obtained with the other meshes. Using the medium mesh, only the free surface model attains results as good as those obtained with the fine mesh. The enriched pressure two-phase model produces some distortion of the interface. Using the normal two-phase model the solution obtained on the medium and coarse meshes is so bad that the interface has disappeared from the mesh at $t=100 \mathrm{~s}$ and therefore no results are shown. Even with the coarse mesh the free surface model manages to 

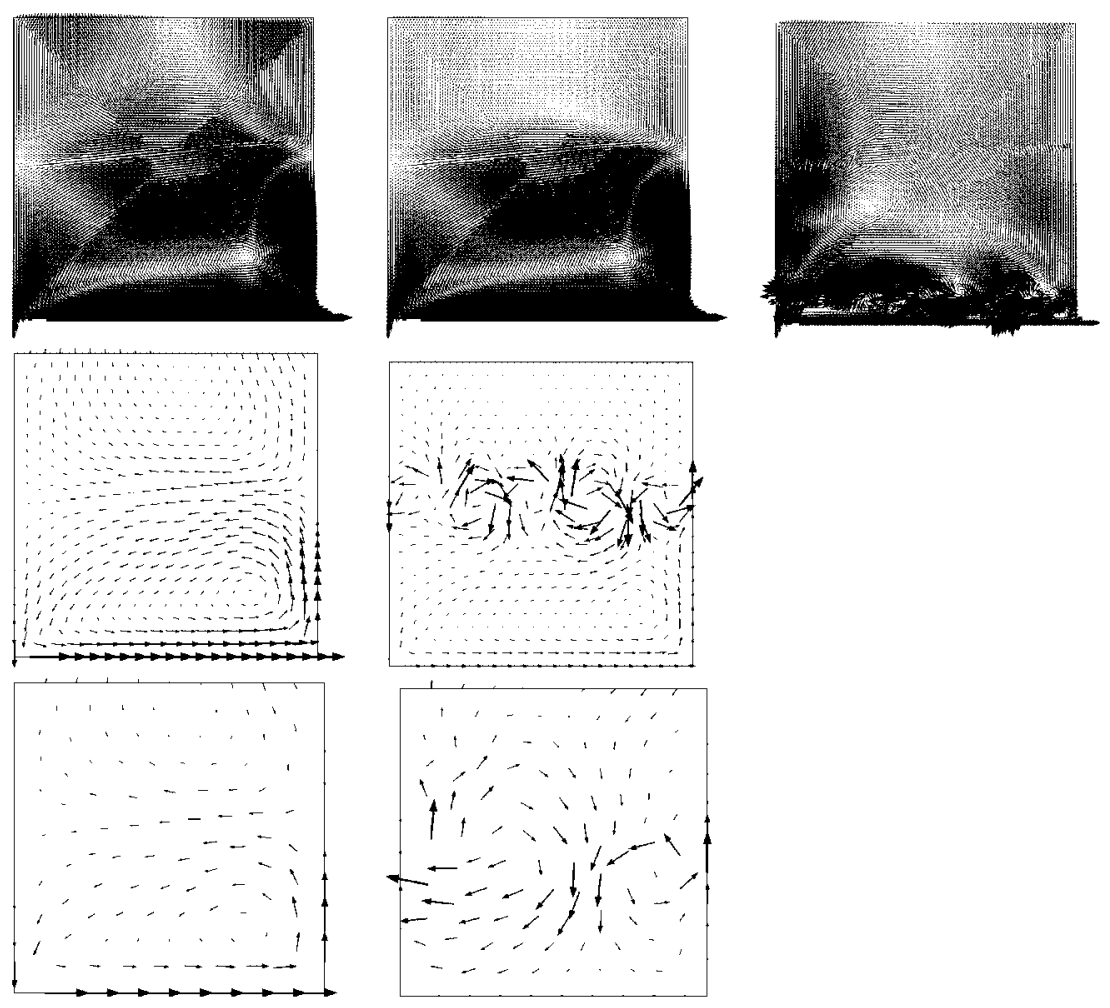

Free Surface

Enriched pressure FM-ALE model two-phase model

Normal two-phase model

Figure 4. Flow pattern for the different meshes and numerical models at $t=100 \mathrm{~s}$ (top: very fine mesh, middle: medium mesh, bottom: coarse mesh).

obtain the correct interface position. With the enriched pressure two-phase model an important mass loss can be observed when using the coarse mesh.

The flow pattern is compared in Figure 4. Again, using the finest mesh there is not much difference in the calculated fluid velocities between free surface model and the enriched pressure two-phase model. The velocities drawn in the empty region in the free surface case are simply the velocities used to transport the level set function. With the normal two-phase model, spurious velocities that distort the whole flow field and ruin mass conservation can be observed close to the interface. The free surface model manages to obtain the correct flow field both with the medium and coarse meshes. With the enriched pressure two-phase model, spurious velocities are obtained close to the interface with the medium mesh. They increase in the coarse mesh. For the normal two-phase model no results are shown for the medium and coarse meshes because, as we have already mentioned, the fluid has disappeared at $t=100 \mathrm{~s}$. It is interesting to point out that the normal two-phase model produces such bad results because we have chosen a low Froude number case. When the Froude number raises all three models give acceptable results. 

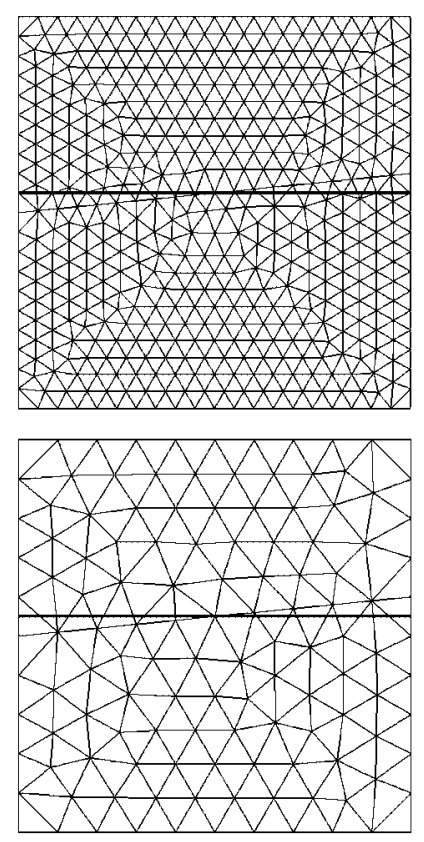

Flow field

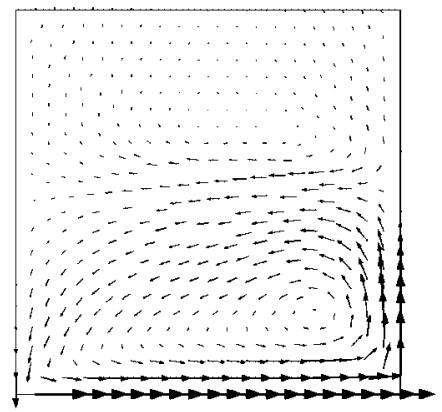

Medium

mesh

Coarse

mesh

Figure 5. Flow field and interface position at $t=100 \mathrm{~s}$ using the Eulerian free surface model.

The results obtained with the Eulerian simplified free surface model have not been shown up to now because they are very similar to those obtained with the FM-ALE free surface model. They are presented in Figure 5 for the medium and coarse meshes so that they can be compared with the results shown previously (Figures 3 and 4) for the FM-ALE model. In the rest of the examples presented in this paper both free surface models have always given nearly the same results and therefore only the results obtained with the FM-ALE model will be shown.

\subsection{Three-dimensional vertical channel}

The second example is a $20 \mathrm{~m}$ high vertical channel with a square cross section (side length $L=5 \mathrm{~m}$ ). The channel is fed from the bottom with a heavier fluid at a constant (both in space and time) $1 \mathrm{~m} / \mathrm{s}$ velocity and the upper face is left free so that the lighter fluid can escape. No friction is assumed on the walls. The initial interface is flat and at $2.5 \mathrm{~m}$ from the entrance. The solution for this problem is very simple. The velocity in the whole mesh, included the interface, should be equal to the inlet velocity and the interface should remain flat.

A three-dimensional unstructured tetrahedral mesh with 1106 nodes and 4921 elements is used. The material properties used (SI units) are $\rho_{1}=1000, \mu_{1}=100$ for the fluid on the bottom, and (for the two-phase models) $\rho_{2}=10, \mu_{2}=1$ for the one on top. The time step size is $0.1 \mathrm{~s}$. The Reynolds number based on the length of the square section is $R e=50$ and the Froude number $F r=0.02$. 

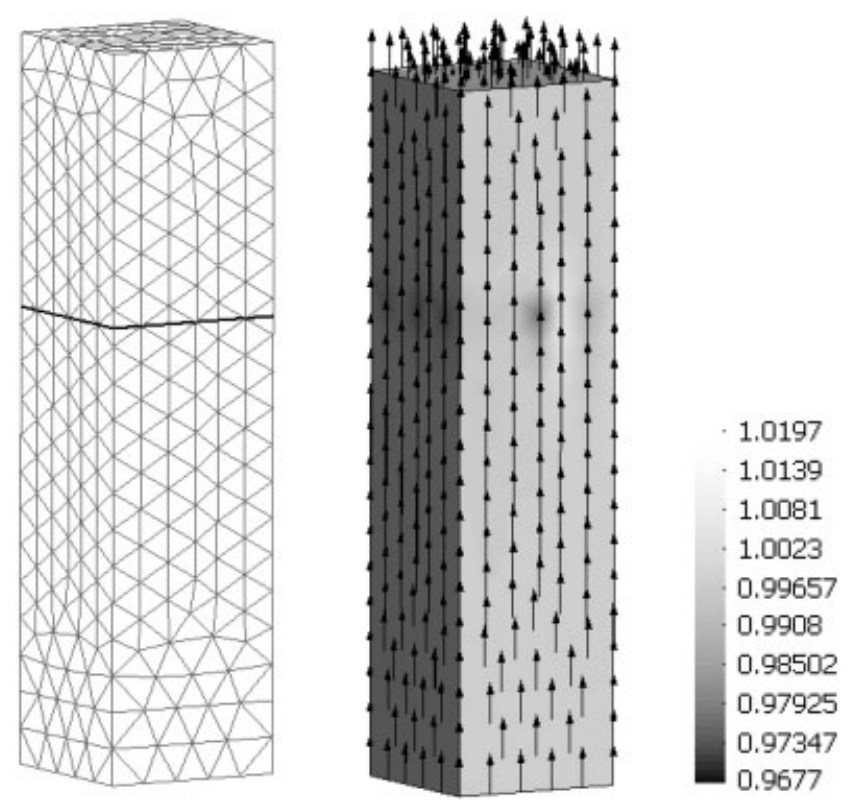

Figure 6. Interface shape and vertical velocity band plot together with velocity vectors using the FM-ALE free surface model.

In Figures 6 and 7 we show the shape of the interface and the velocity field obtained with the free surface FM-ALE model proposed in this paper and with the enriched pressure two-phase model proposed in [11]. Both models provide the correct solution; the interface remains flat and its displacement corresponds to the amount of injected fluid. When the normal two-phase flow model is used (see Figure 8) the results are very poor. The velocity shows important oscillations close to the interface and there is an important mass loss. The mass loss is so important that the free surface remains nearly at its initial height. The source of the errors in the normal two-phase flow model is the impossibility of the shape functions to capture the discontinuous pressure gradient that exists at the interface. One way to solve the problem is to enrich the pressure shape functions so as to represent the discontinuous pressure gradient more accurately. The other way to solve the problem is to use a free surface model and thus avoid the existence of a discontinuous pressure gradient by modelling only one fluid. The slight errors that remain in the velocity field with the two successful models can be attributed to the fact that the numerical resolution of the level set is not exact and therefore the deviations in the shape of the interface give rise to small variations in the velocity.

\subsection{Sloshing problem}

As a final example, we consider a sloshing problem. It is a simple problem of free oscillation of an incompressible liquid in a container. Following [8-10] we consider a liquid column of width $b$ with an initial surface profile corresponding to the first antisymmetric mode of vibration and a null initial velocity. The height of the free interface is

$$
\eta(x, 0)=1.0+a \sin \frac{\pi}{b} x
$$



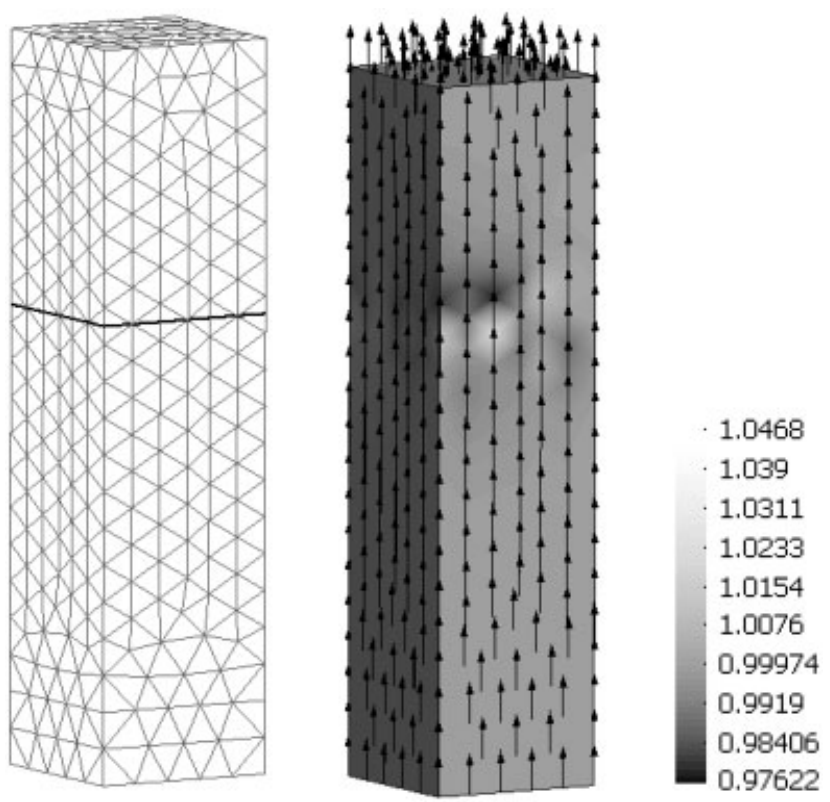

Figure 7. Interface shape and vertical velocity band plot together with velocity vectors using the two-phase model with pressure enrichment.
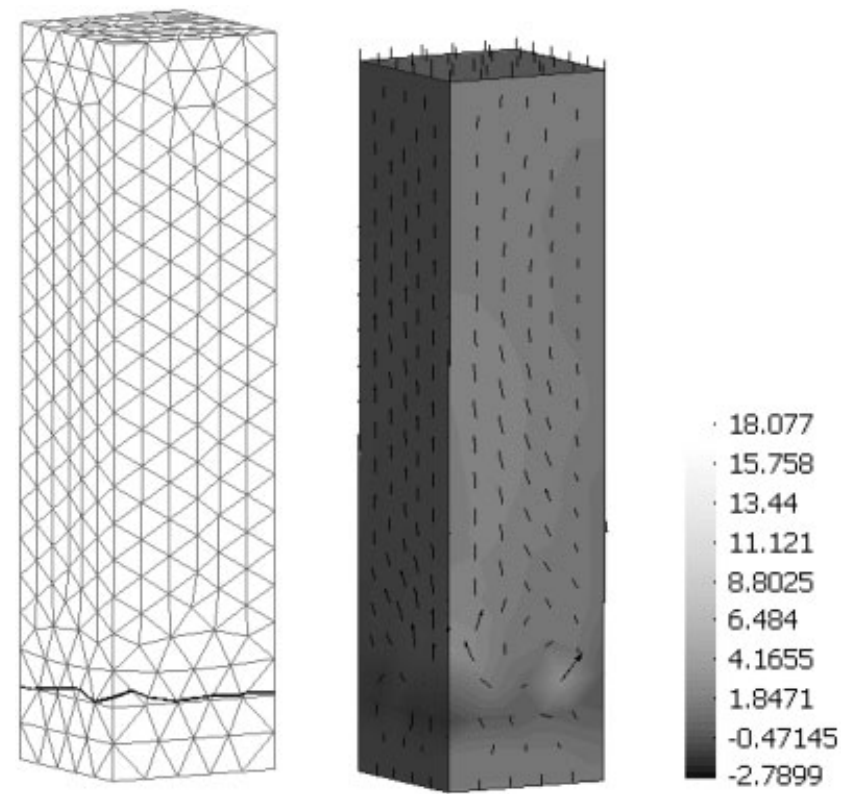

Figure 8 . Interface shape and vertical velocity band plot together with velocity vectors using the normal two-phase model. 


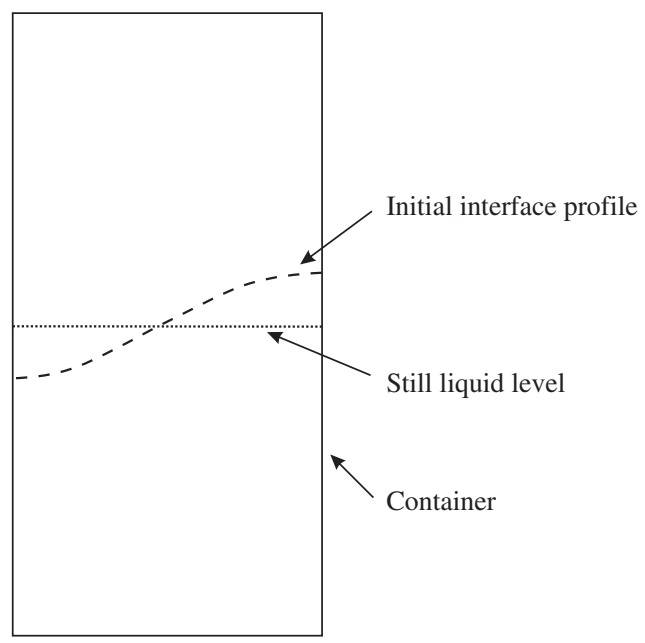

Figure 9. Diagram for the sloshing problem.
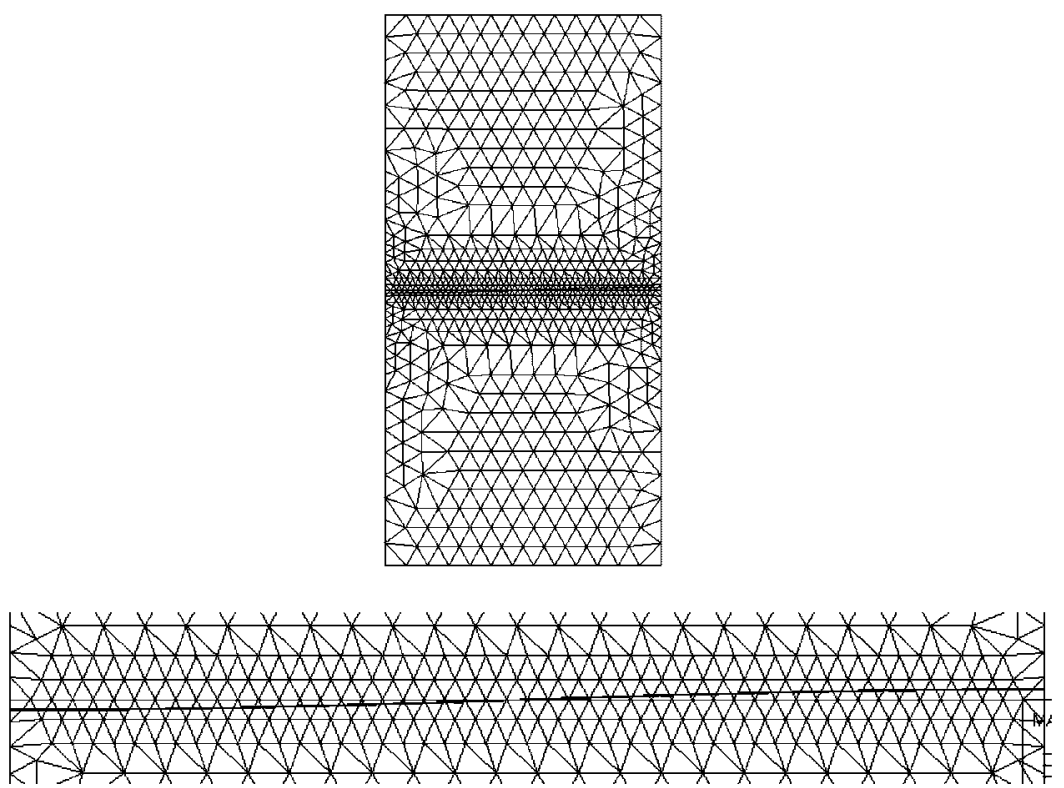

Figure 10. Mesh and initial interface position for the sloshing problem. Top: general view. Bottom: detail.

where $a$ is the amplitude of oscillation. Thus, the initial condition for the level set function is

$$
\psi(x, y, 0)=\eta(x, 0)-y
$$

where $x=0$ at the middle of the container and $y=0$ at the bottom (Figure 9). 
Using the same parameters as in the cited papers, the amplitude is taken as $a=0.01$ and the kinematic viscosity as $v=0.01$. In the results obtained with the two-phase model (with or without enrichment) the density and dynamic viscosity of the second fluid are 100 times smaller than those of the bottom one so that it does not affect significantly the flow of the heavier fluid. The container walls are assumed to be impermeable and allow for free slip. The mesh we use has a width $b=1$ and a height $h=2$ and is filled with fluid only in the lower half (see Figure 10). The mesh has 1394 triangular elements and 747 nodes and is refined close to the interface.

In Figure 11 we show the position of the interface after $11 \mathrm{~s}$, using: (1) the free surface FM-ALE model; (2) the two-phase enriched pressure model and (3) the normal two-phase model. Figure 12 shows the computed time history of $\eta(b / 2, t)$ for the three cases together with the results presented by Ramaswamy et al. [9] Both figures show that using the normal two-phase model there is a

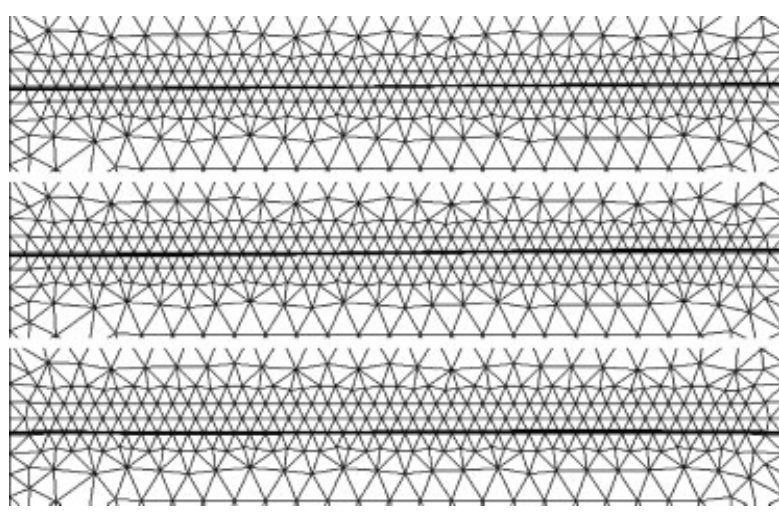

Free surface

FM-ALE model

Enriched

pressure two

phase model

Normal two

phase model

Figure 11. Interface position at $t=11 \mathrm{~s}$ for the sloshing problem.

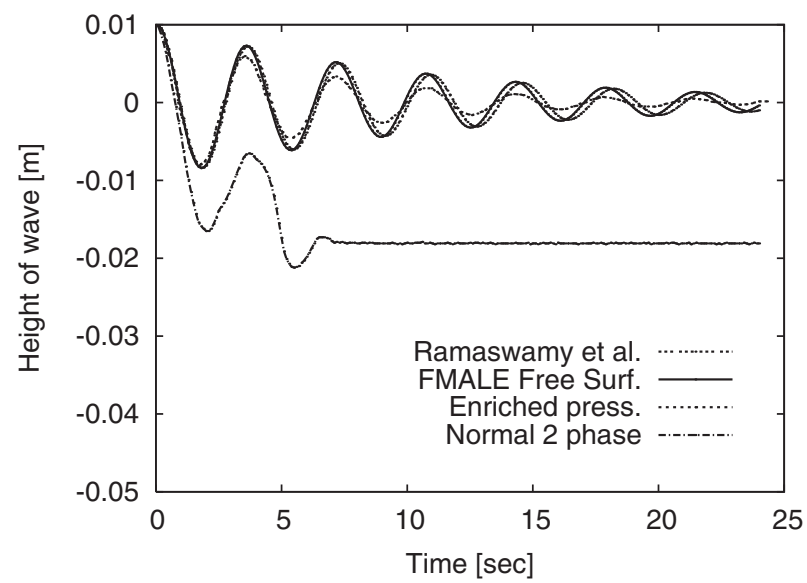

Figure 12. Time histories of surface elevation amplitude for the sloshing problem. 
significant mass loss. The results obtained with the free surface and enriched pressure two-phase models agree closely with the ones reported by Ramaswamy et al. [9]. In the normal two-phase model case there is a significant mass loss until the interface aligns with element faces. Then the mass loss stops because the pressure gradient can be represented satisfactorily but the results have lost any meaning.

Some slight differences can be observed in the phase and amplitude obtained with the FM-ALE model, the two-phase enriched pressure model and the results obtained by Ramaswamy et al. [9]. These differences can be attributed to the fact that the mesh used is different to the one used by Ramaswamy and co-workers or to the particularities of the numerical method, such as the stabilization technique that has been used. It is interesting to note that the amplitude obtained with the FM-ALE model and the two-phase enriched pressure model is slightly higher than the one obtained by Ramaswamy and co-workers suggesting that our numerical method introduces less artificial diffusion than the one used by Ramaswamy et al.

\section{CONCLUSIONS}

In this paper we have introduced a formulation for free surface flows on fixed meshes. The key difference with typical Eulerian formulations for free surface flows is that we do not solve the Navier-Stokes equations on the whole mesh. Taking advantage of the fact that we have a boundary condition at the free surface we can model the flow only in the fluid region. By doing so we are able to avoid the difficulty of modelling a discontinuous pressure gradient in the cut elements. The use of enhanced integration allows us to impose the Neumann boundary condition at a surface that does not coincide with element faces quite simply. On the other hand, the model differs from ALE or Lagrangian simulations on the fact that we use a fixed mesh. Two versions of the model have been proposed, one that uses an ALE formulation on fixed meshes (FM-ALE) and the other one that is more closely related to Eulerian formulations. No significant difference has been observed between the numerical results obtained with the two versions.

The FM-ALE version calculates the velocities in the empty region only to transport the level set function. The other version also uses the velocities in the empty region to model the transient term in nodes that go from the empty region to the filled one in a way that mimics what happens in a two-phase flow with negligible properties in the second fluid. The model takes into account the fact that in a free surface flow the velocities in the fluid region influence the velocities in the empty region but not the other way round.

The numerical results have shown that the formulation can provide very satisfactory results for low Froude number flows where the typical two-phase flow model fails. Compared with the two-phase model with enriched pressure [11] the free surface model has produced equal or better results in all the analysed cases.

The free surface model is simpler than the enriched pressure two-phase model because no enrichment is needed. On the other hand, since the flow equations are solved in the liquid and the empty region separately, the computational cost is reduced.

The examples shown in this paper demonstrate that the free surface model can introduce significant improvements compared with a typical two-phase flow finite element model. It allows to avoid spurious velocities and enhances mass conservation. 


\section{ACKNOWLEDGEMENTS}

A. H. Coppola-Owen would like to acknowledge the support received from the Departament d'Universitats, Recerca i Societat de la Informació of the Generalitat de Catalunya (Catalan Government) and the European Social Fund through a doctoral grant.

\section{REFERENCES}

1. Chang YC, Hou TY, Merriman B, Osher S. A level set formulation of Eulerian interface capturing methods. Journal of Computational Physics 1996; 124:449-464.

2. Sussman M, Almgren AS, Colella JB, Howell LH, Welcome ML. An adaptive level set approach for incompressible two phase flows. Journal of Computational Physics 1999; 148:81-124.

3. Osher S, Fedkiw RP. Level Set Methods and Dynamic Implicit Surfaces. Springer: Berlin, 2003.

4. Thompson E. Use of the pseudo-concentration to follow creeping viscous during transient analysis. International Journal for Numerical Methods in Engineering 1986; 6:749-761.

5. Hirt CW, Nichols BD. Volume of fluid (VOF) method for the dynamics of free boundaries. Journal of Computational Physics 1981; 39:201-225.

6. Codina R, Schäfer U, Oñate E. Mould filling simulation using finite elements. International Journal of Numerical Methods for Heat and Fluid Flow 1994; 4:291-310.

7. Lewis RW, Usmani AS, Cross JT. Efficient mould filling simulation in metal castings by an explicit finite element method. International Journal for Numerical Methods in Engineering 1995; 20:493-506.

8. Ramaswamy B. Numerical simulation of unsteady viscous free surface flow. Journal of Computational Physics 1990; 90:396-430.

9. Ramaswamy B, Kawahara M, Nakayama T. Lagrangian finite element method for the analysis of two-dimensional sloshing problems. International Journal for Numerical Methods in Fluids 1985; 6:659-670.

10. Radovitzky R, Ortiz M. Lagrangian finite element analysis of Newtonian fluid flows. Journal of Computational Physics 1998; 43:607-619.

11. Coppola-Owen AH, Codina R. Improving Eulerian two-phase flow finite element approximation with discontinuous gradient pressure shape functions. International Journal for Numerical Methods in Fluids 2005; 49:1278-1304.

12. Carrica PM, Wilson RV, Stern F. An unsteady single-phase level set method for viscous free surface flows. International Journal for Numerical Methods in Fluids, in press.

13. Codina R. A stabilized finite element method for generalized stationary incompressible flows. Computer Methods in Applied Mechanics and Engineering 2001; 190:2681-2706.

14. Brezzi F, Fortin M. Mixed and Hybrid Finite Element Methods. Springer: Berlin, 1991.

15. Houzeaux G, Codina R. A finite element model for the simulation of lost foam casting. International Journal for Numerical Methods in Fluids 2004; 46:203-226.

16. Houzeaux G, Codina R. Transmission conditions with constraints in finite element domain decomposition methods for flow problems. Communications in Numerical Methods in Engineering 2001; 17:179-190.

17. Sethian JA, Smereka P. Level set methods for fluid interfaces. Annual Review of Fluid Mechanics 2003; 35:341-372. 